\title{
Computing the optical near-field distributions around complex subwavelength surface structures: A comparative study of different methods
}

\author{
Dominique Barchiesi \\ Laboratoire d'optique P.M. Duffieux, URA CNRS 214, Université de Franche Comté, 25030 Besançon, France \\ Christian Girard \\ Laboratoire de Physique Moléculaire, URA CNRS 772, Université de Franche Comté, 25030 Besançon, France \\ Olivier J. F. Martin \\ Swiss Federal Institute of Technology, Eidgenössische Technische Hochschule-Zentrum, 8092 Zurich, Switzerland \\ Daniel Van Labeke and Daniel Courjon \\ Laboratoire d'optique P.M. Duffieux, URA CNRS 214, Université de Franche Comté, 25030 Besançon, France
}

(Received 20 February 1996)

\begin{abstract}
Some 15 years ago, optical topographic signals with subwavelength resolution were obtained independently by several experimental teams. Since this exploratory period, a growing number of experimental configurations have been proposed and continuously developed. Simultaneously, this research field was supported by different theoretical works, aimed at developing our understanding of the interaction of optical fields with mesoscopic objects. Over the past three years, several theoretical frameworks have been proposed (Green's functions, field susceptibility, boundary conditions methods, multiple multipoles expansions, etc.). In this paper, an attempt at a careful comparison between two classes of numerical models is presented. Using the same test object, we discuss and compare the numerical solutions issued from a reciprocal-space perturbative method (Rayleigh approximation) and the solution originating from a direct-space integral approach (Green's function or field susceptibility). The discussion is given for different values of the relevant experimental parameters. The convergence of both approaches is investigated. [S1063-651X(96)07309-6]

PACS number(s): 42.25.-p, 61.16.Ch, 02.60.Cb, 02.70.-c
\end{abstract}

\section{INTRODUCTION}

When an optical electromagnetic wave interacts with a localized surface defect, the electromagnetic energy distribution observed around the surface defect is extremely sensitive both to the illumination mode and the physical parameters of the defect (shape, optical index, and relative size compared to the wavelength) [1-5].

A detailed understanding of this optical interaction between subwavelength structures and external light sources represents indisputably one of the most serious challenges raised by the tremendous recent experimental progress of near-field optics (NFO) [6,7].

The accurate description of the optical field distribution, prior to its local detection, is mandatory for describing properly the image formation mechanisms in NFO [8]. The main difficulties in achieving this goal are inherent to the complexity of the geometries investigated in NFO (nonperiodic objects, localized surface defects, nanometer size holes, etc.) as well as to the need of accounting for a large spectra of nonradiative optical field components. Particularly, corners, sharp edges, and angular regions much smaller than the incident wavelength generate specific difficulties for most of the theoretical schemes and numerical methods for solving Maxwell's equations. Recently, Van Labeke and Barchiesi proposed a brief overview of the main numerical methods currently applied to NFO [9]. During the last three years this research field has grown exponentially and generated an important amount of peculiar results obtained with different methods [10-22] (Green functions, field susceptibilities, boundary conditions based method, multiple multipoles description, etc.). Nowadays, in order to guide the ongoing development of NFO instrumentation, and to improve the predictive character of current computerized work, we feel that it is necessary to assess the validity range as well as the versatility of different model classes.

In this paper an attempt at a careful comparison between two classes of numerical approaches is presented. By using the same test object, we discuss and compare the numerical solution obtained from a reciprocal-space perturbative approach based on Rayleigh approximation [12,18] and the solution obtained with a direct-space macroscopic approach based on the Green's function technique or fieldsusceptibility technique $[10,11,16,20]$. A discussion is given for different values of the external parameters accessible to most experimental setups (polarization and direction of the incident light, observation distance).

The paper is organized as follows. In Sec. II we define the geometry of our test case. The reciprocal-space approach is detailed in Sec. III and the direct-space approach in Sec. IV. In Sec. V we compare the results obtained with these two approaches and analyze the convergence of each method. Finally, we conclude and discuss the applicability limits of both approaches in Sec. VI. 

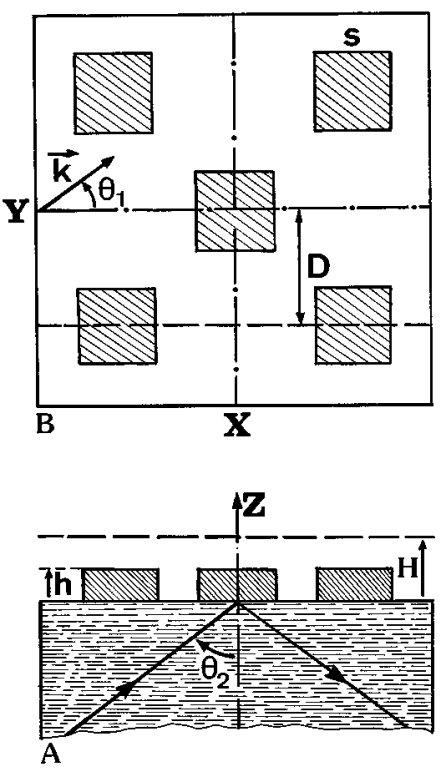

FIG. 1. Three-dimensional system considered in our simulations: (A) side view and (B) top view. This system is composed of five identical pads of square section, placed on a perfectly flat surface, at the nodes of a centered square lattice. The sample is illuminated by total internal reflection and the resulting field distribution is computed in different planes $Z=H$ above the substrate. The illumination mode is defined by two angular parameters: the incident angle $\theta_{2}$ defined with respect to the normal axis $O z$ and the angle $\theta_{1}$ that defines the direction of the evanescent surface wave traveling along the surface.

\section{TEST OBJECT AND ILLUMINATION MODE}

As test object, let us consider the three-dimensional surface protrusion depicted in Fig. 1. This object is formed by five square pads placed on a perfectly flat surface. The optical index of this protrusion is similar to the surface index $(n=1.5)$. The upper half space is air $(n=1)$.

We consider the total internal reflection configuration (TIR), where the surface is illuminated from below by a monochromatic plane wave of frequency $\omega_{0}$, incident at an angle $\theta_{2}$ larger than the total reflection angle $\theta_{\text {tot }}$. Note that the present treatment could also be applied to other experimental configurations, based, for example, on external reflection. Two different incident polarizations can be considered: $s$ polarization, where the incident electric field is parallel to the glass-air interface, and $p$ polarization, where it is in the plane of incidence (Fig. 1).

As a total reflection occurs at the glass-air interface, only an evanescent electrical field $\mathbf{E}_{0}(\mathbf{r}, t)$ is established above the surface $(z>0)$. If we neglect the protrusion and consider a perfectly flat surface, this evanescent field can be obtained analytically. This analytical solution plays a key role in the two procedures investigated in this paper. Indeed, for both procedures, the final solution of the problem is obtained from this zeroth-order solution:

$$
\mathbf{E}_{0}(\mathbf{r}, t)=\mathbf{E}_{0}(\mathbf{r}) e^{-i \omega_{0} t}=\mathbf{E}_{\mathbf{0}} e^{i \mathbf{k} \cdot l} e^{-K z} e^{-i \omega_{0} t},
$$

where $\mathbf{r}=(x, y, z)=(\boldsymbol{l}, z)$ represents an observation point above the surface,

$$
K=\frac{\omega_{0}}{c}\left(\sin ^{2} \theta_{2}-\sin ^{2} \theta_{\text {tot }}\right)^{1 / 2},
$$

and

$$
\|\mathbf{k}\|=\frac{\omega_{0}}{c} \sin \theta_{2}
$$

When, for example, the incident field is propagating along the $O y$ axis (which corresponds to $\theta_{1}=\pi / 2$ in Fig. 1), Eqs. (1), (2), and (3) lead to

$$
E_{0 x}(\mathbf{r})=A_{0} T_{s}, \quad E_{0 y}(\mathbf{r})=0, \quad E_{0 z}(\mathbf{r})=0,
$$

for $s$-polarized illumination and

$$
E_{0 x}(\mathbf{r})=0, \quad E_{0 y}(\mathbf{r})=A_{0} T_{p} \delta_{c}, \quad E_{0 z}(\mathbf{r})=A_{0} T_{p} \delta_{s},
$$

for $p$-polarized illumination, where

$$
\delta_{s}=\frac{\sin \left(\theta_{2}\right)}{\sin \left(\theta_{\mathrm{tot}}\right)}, \quad \delta_{c}=\frac{i K}{k_{0} \sin \left(\theta_{\mathrm{tot}}\right)}
$$

In Eqs. (4) and (5), $A_{0}$ is an arbitrary amplitude that depends on the illumination intensity, and the factors $T_{s}$ and $T_{p}$ are the usual transmission coefficients for each polarization [23].

\section{RECIPROCAL-SPACE PERTURBATIVE METHOD (RSPM)}

When a surface exhibits well-defined protrusions, different theoretical schemes may be applied to describe its optical response to an arbitrary excitation. As extensively discussed in [3-5], the perturbative diffraction method provides an interesting framework to study low relief objects. In this case, the boundary conditions problem is solved by assuming that the fluctuation heights of the surface are weak compared to the incident wavelength. Corrections to the usual result associated with a perfectly flat sample are obtained in terms of the Fourier transform of the corrugation function $\xi(l)$ limiting the surface of the solid (cf. Fig. 2). This method represents an interesting tool towards the interpretation of NFO images and does not need extensive computational work. Nevertheless, we must emphasize that such a perturbative approximation limits its validity range to surface corrugations of weak amplitude. We give in this section a brief description of the main features of this method.

The zeroth-order field $\mathbf{E}_{0}(\mathbf{r})$ above the sample (i.e., in the absence of corrugation) is defined in the preceding section [Eq. (1)]. The additional contribution $\mathbf{E}_{d}(\mathbf{r})$ introduced by the surface protrusion (the so-called diffracted field) is determined by assuming the following plane wave expansion:

$$
\mathbf{E}_{d}(\mathbf{r})=\int \widetilde{\mathbf{E}}_{d}(\mathbf{q}) e^{i(\mathbf{q} \cdot \boldsymbol{l}+v z)} d \mathbf{q}
$$

where $\mathbf{q}=\left(q_{x}, q_{y}\right)$ and $v$ represents the Cartesian components of the different wave vectors associated to the diffracted surface field. These components verify the wellknown dispersion equation 


$$
q_{x}^{2}+q_{y}^{2}+v^{2}=\frac{\omega_{0}^{2}}{c^{2}}=k_{0}^{2},
$$

and the twofold integral in Eq. (7) runs over the spatial frequencies $\left(q_{x}, q_{y}\right)$ and generally contains both homogeneous (propagating) and inhomogeneous (evanescent) waves. A given plane wave of the series is homogeneous if the component $v$ is real (i.e., $q_{x}^{2}+q_{y}^{2} \leqslant \omega_{0}^{2} / c^{2}$ ) or evanescent if $v$ is a pure imaginary number (i.e., $q_{x}^{2}+q_{y}^{2}>\omega_{0}^{2} / c^{2}$ ). Note that the amount of evanescent components is directly related to the lateral size of the surface protrusion with respect to the incident wavelength $\lambda_{0}$. Thus, when we deal with a sample that displays topographical details much smaller than $\lambda_{0}$, the Fourier integral (7) is mainly composed by evanescent components and the resulting diffracted field $\mathbf{E}_{d}(\mathbf{r})$ turns out to be confined around the surface roughness.

The evaluation of the field amplitude $\widetilde{\mathbf{E}}_{d}(\mathbf{q})$ requires the Fourier transform of the topographical function $\xi(l)$ :

$$
\xi(\boldsymbol{l})=\int \widetilde{\xi}(\mathbf{q}) e^{i \mathbf{q} \cdot l} d \mathbf{q}
$$

The application of standard boundary conditions at the surface $z=\xi(l)$ leads to complex relations between the incident and the diffracted field. This difficulty may be avoided by working within the perturbative approximation introduced some years ago by Elson [3], Toigo et al. [4], and Agarwal [5] to study the reflectivity of metallic corrugated surfaces. This approximation consists in expanding the exponential function occurring in Eq. (7) as a power series of $v \xi(l)$ :

$$
e^{i[\mathbf{q} \cdot \boldsymbol{l}+v \xi(\boldsymbol{l})]}=e^{i \mathbf{q} \cdot \boldsymbol{l}}\left\{1+v \xi(\boldsymbol{l})+[v \xi(\boldsymbol{l})]^{2}+\cdots\right\} .
$$

As discussed in [3-5], the validity range of this expansion depends on the $v \xi(\boldsymbol{l})$ amplitude and therefore must be restricted to weak surface height fluctuations compared to the incident wavelength. For small surface structure heights, this expansion can be limited to the first-order term. In this case, the diffracted amplitude is proportional to the Fourier transform $\widetilde{\xi}(\mathbf{q})$ of the surface profile and depends linearly on the zeroth-order field $\mathbf{E}_{0}(\mathbf{r})$ associated with a perfectly planar sample:

$$
\widetilde{\mathbf{E}}_{d}(\mathbf{q})=i\left(n^{2}-1\right) \widetilde{\xi}(\mathbf{q}-\mathbf{k}) \widetilde{\mathbf{A}}(\mathbf{q}) \cdot \mathbf{E}_{0}(\mathbf{r})
$$

The $(3 \times 3)$ transfer matrix $\widetilde{\mathbf{A}}(\mathbf{q})$ in Eq. (11) is defined by

$$
\widetilde{\mathbf{A}}(\mathbf{q})=\frac{k_{0}^{2}}{v+v^{\prime}} \mathbf{I}-\widetilde{\mathbf{B}}(\mathbf{q}),
$$

where I represents the identity matrix, $v^{\prime}$ a $z$ component of the wave vector inside the sample $\left(v^{\prime 2}=\mathbf{q}^{2}-n^{2} k_{0}^{2}\right)$, and

$$
\widetilde{\mathbf{B}}(\mathbf{q})=\frac{1}{v^{\prime}+n^{2} v}\left(\begin{array}{ccc}
q_{x}^{2} & q_{x} q_{y} & q_{x} v \\
q_{x} q_{y} & q_{y}^{2} & q_{y} v \\
v^{\prime} q_{x} & v^{\prime} q_{y} & v^{\prime} v
\end{array}\right) .
$$

By combining the above results [Eqs. (7), (11), (12), and (13)], we can now derive the total optical field generated near the surface protrusions:
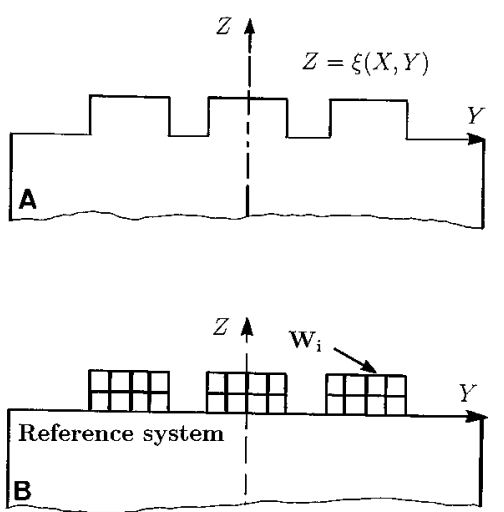

FIG. 2. Schematic illustration of the discretization principle underlying each numerical method investigated in the present study. (A) For the reciprocal-space perturbative method (RSPM), the matching of the different Fourier components is realized on the entire boundary $Z=\xi(X, Y)$ of the object. (B) In the direct-space integral equation method (DSIEM), only the geometry elements that do not belong to the reference system (i.e., the pads above the surface, the latest being the reference system) must be discretized into meshes of volume $W_{i}$.

$$
\begin{aligned}
\mathbf{E}(\mathbf{r})= & \mathbf{E}_{0}(\mathbf{r})+\int i\left(n^{2}-1\right) e^{i(\mathbf{q} \cdot l+v z)} \widetilde{\xi}(\mathbf{q}-\mathbf{k}) \\
& \times \widetilde{\mathbf{A}}(\mathbf{q}) \cdot \widetilde{\mathbf{E}}_{0}(\mathbf{q}) d \mathbf{q} .
\end{aligned}
$$

This field can be evaluated numerically with standard fast fourier transform (FFT) routines. At this stage, we note that in this reciprocal-space scheme, the structural information on the object is given by the Fourier transform of the surface profile $\widetilde{\xi}(\mathbf{q}-\mathbf{k})$. Consequently, the accuracy of the results will directly depend on the number of spatial harmonics used in the FFT. The presence of a second corrugated interface could be introduced without any formal difficulty by extending the present method to a multilayered system [18].

Let us emphasize that the RSPM is based on the discretization of the entire surface profile $\xi(X, Y)$, as illustrated in Fig. 2(A).

\section{DIRECT-SPACE INTEGRAL EQUATION METHOD (DSIEM)}

The numerical difficulties inherent to the low symmetry of the subwavelength objects currently analyzed with SNOM devices may be overcome by solving directly the integral equation associated with Maxwell's equations $[10,11,16,19,20]$. The kernel of the master equation can then be factorized in two different ways, leading to two different kinds of dyadic propagators: field susceptibility or Green's tensor. This technique, associated with a convenient discretization procedure, has proven to be powerful and versatile for studying many properties associated with local probe experiments.

In Sec. III we have seen that the RSPM solution is composed of two terms: the zeroth-order field $\mathbf{E}_{0}(\mathbf{r})$ representing the solution for a perfectly flat surface and the field $\mathbf{E}_{d}(\mathbf{r})$ diffracted by the protrusions. The DSIEM also relies on the splitting of the solution into two terms, based on the decomposition of the system into two parts: a highly symmetrical 
reference system (in the simulations to be discussed below, a perfect planar surface limiting the infinite homogeneous half spaces, Fig. 1), and a low symmetry, spatially limited system placed in interaction with this reference system [the threedimensional (3D) defects lying on the surface in Fig. 1].

Starting from the zeroth-order field $\mathbf{E}_{0}(\mathbf{r})$ defined in Sec. II, it is a simple matter to derive the self-consistent field $\mathbf{E}(\mathbf{r})$ by introducing the implicit integral equation $[10,11,19,20]$

$$
\mathbf{E}(\mathbf{r})=\mathbf{E}_{0}(\mathbf{r})+\int \mathbf{K}\left(\mathbf{r}, \mathbf{r}^{\prime}\right) \cdot \mathbf{E}\left(\mathbf{r}^{\prime}\right) d \mathbf{r}^{\prime} .
$$

In this self-consistent equation, the integral runs over the volume occupied by the surface protrusions.

Depending on the adopted physical point of view, the dyadic kernel $\mathbf{K}\left(\mathbf{r}, \mathbf{r}^{\prime}\right)$ in Eq. (15) may be factorized in two equivalent ways.

(i) In the framework of the field-susceptibility method, this factorization reads [10]

$$
\mathbf{K}\left(\mathbf{r}, \mathbf{r}^{\prime}\right)=\mathbf{S}\left(\mathbf{r}, \mathbf{r}^{\prime}\right) \cdot \chi\left(\mathbf{r}^{\prime}\right),
$$

where $\mathbf{S}\left(\mathbf{r}, \mathbf{r}^{\prime}\right)$ represents the field susceptibility of the reference system and $\boldsymbol{\chi}\left(\mathbf{r}^{\prime}\right)$ the linear susceptibility of the perturbation (localized defect). In the case of continuous matter, $\boldsymbol{\chi}\left(\mathbf{r}^{\prime}\right)$ is given in terms of the local optical index $n$ associated with the surface defect (cgs units)

$$
\boldsymbol{\chi}\left(\mathbf{r}^{\prime}\right)=\frac{n^{2}\left(\mathbf{r}^{\prime}\right)-1}{4 \pi} \mathbf{I}
$$

for all the points $\mathbf{r}^{\prime}$ in the localized defect, and

$$
\chi\left(\mathbf{r}^{\prime}\right)=0
$$

outside the defect. This factorization was originally introduced to deal with atoms or molecules adsorbed on a surface [24-26].

(ii) The second point of view uses the Green's dyadic $\mathbf{G}\left(\mathbf{r}, \mathbf{r}^{\prime}\right)$ of the reference system to factorize the kernel of Eq. (15):

$$
\mathbf{K}\left(\mathbf{r}, \mathbf{r}^{\prime}\right)=\mathbf{G}\left(\mathbf{r}, \mathbf{r}^{\prime}\right) \cdot \mathbf{V}\left(\mathbf{r}^{\prime}\right)
$$

where $\mathbf{V}\left(\mathbf{r}^{\prime}\right)$ is the dyadic defined by

$$
\mathbf{V}\left(\mathbf{r}^{\prime}\right)=-k_{0}^{2} \frac{n^{2}\left(\mathbf{r}^{\prime}\right)-1}{4 \pi} \mathbf{I}
$$

for all points $\mathbf{r}^{\prime}$ located inside the surface defect, and

$$
\mathbf{V}\left(\mathbf{r}^{\prime}\right)=\mathbf{0}
$$

outside the perturbation. This factorization is standard in classical electrodynamics theory [27]. Moreover, these two different factorizations of the kernel of Eq. (15) emphasize that the DSIEM formalism is aimed at treating discrete as well as continuous perturbations. Therefore the transition from classical to quantum electrodynamics problems can be performed in the same unique framework [28].

Whereas the RSPM relies on the discretization of the entire surface profile, the DSIEM requires only the discretiza- tion of the geometrical elements that do not belong to the reference system, as illustrated in Fig. 2(B).

Dividing the surface defect into $N$ meshes of respective volume $W_{i}$, centered at $\mathbf{r}_{i}, i=1, \ldots, N$; and introducing the discretized variables $\mathbf{E}_{i}, \mathbf{E}_{i}^{0}$, and $\mathbf{K}_{i, j}$ for $\mathbf{E}\left(\mathbf{r}_{i}\right), \mathbf{E}_{0}\left(\mathbf{r}_{i}\right)$, and $\mathbf{K}\left(\mathbf{r}_{i}, \mathbf{r}_{j}\right)$, respectively, we obtain the discretized form of Eq. (15):

$$
\mathbf{E}_{i}=\mathbf{E}_{i}^{0}+\sum_{k=1}^{N} \mathbf{K}_{i, k} \cdot \mathbf{E}_{k} W_{k}
$$

Equation (22) corresponds to a large system of algebraic equations that must be solved to obtain the self-consistent solution $\mathbf{E}(\mathbf{r})$ for a given incident field $\mathbf{E}_{\mathbf{0}}(\mathbf{r})$. It is important to note that for physical systems relevant to NFO, the condition number of the matrix associated with Eq. (22) can become very large; therefore extremely stable algorithms must be used to solve this system of equations [19].

The diagonal elements $\mathbf{K}_{i, i}$ of the discretized kernel play an extremely important role in this formalism. Indeed, they account for the local response of the matter to the external field (depolarization) and must be handled with the appropriate renormalization procedure [29].

\section{COMPARISON OF THE METHODS}

Let us emphasize that the aim of the present paper is not to investigate new physical effects per se, but to show, using an experimentally relevant configuration, that a good agreement between completely different theoretical approaches is obtained. The cross-comparison of the results obtained with such different approaches is important because many fundamental NFO phenomena are extremely subtle and difficult to model. Therefore, in order to efficiently analyze ongoing experimental work, the possible artifacts related to numerical methods must be carefully investigated and the validity domain of each approach assessed.

Our test object is composed of five identical pads of square section, placed on a perfectly flat surface, at the nodes of a centered square lattice (cf. Fig. 1). This structure is defined by three geometrical parameters: the side $s$ and the height $h$ of each individual protrusion and the spacing $D$ between two pads. In the present study, we use $s=40 \mathrm{~nm}$, $D=80 / \sqrt{2} \mathrm{~nm}$ and $h=10 \mathrm{~nm}$, except in Fig. 6, where we use $h=20 \mathrm{~nm}$. The optical indexes of the surface and of the pads are equal $(n=1.5)$ and the surrounding medium is air $(n=1)$. As in the scanning tunneling optical configuration [30-36], the sample is illuminated by total internal reflection and the resulting field distribution is computed in different planes $Z=H$ above the substrate. The illumination mode is defined by two angular parameters: the incident angle $\theta_{2}$ defined with respect to the normal axis $O z$ and the angle $\theta_{1}$ that defines the direction of the evanescent surface wave traveling along the surface. For each incident direction $\left(\theta_{1}, \theta_{2}\right)$, two polarizations can be considered: TE polarization, where the incident electric field vector is parallel to the substrate and TM polarization, where it is in the plane of incidence defined by the vector $\mathbf{k}$. The illumination wavelength in vacuum is $633 \mathrm{~nm}$ and we consider an incident angle $\theta_{2}=45^{\circ}$.

In Fig. 3, we present the total near-field intensity com- 

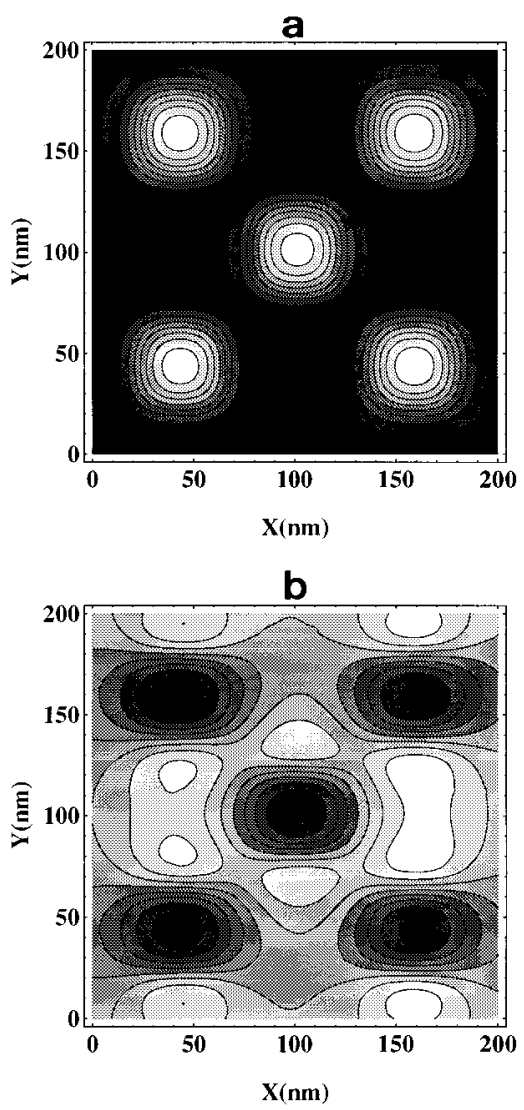

FIG. 3. Total near-field intensity computed at an observation height $H=20 \mathrm{~nm}$, with the RSPM, using 192 harmonics in Eq. (14). High intensity: white; low intensity: black. (a) TM polarization, (b) TE polarization.

puted at an observation height $H=20 \mathrm{~nm}$, with the RSPM, using 192 harmonics in Eq. (14). Two different incident polarizations are considered. For TM polarization, the total electric field intensity reproduces the surface protrusions (the incident field is propagating along $X$ direction) [Fig. 3(a)], whereas for TE polarization, it emphasizes the object's sides orthogonal to the incident field [the incident field is propagating along $X$ direction and polarized along $Y$ direction, Fig. 3(b)]. Therefore, while TM polarization leads to a strong confinement of the total field intensity above the surface defects, TE polarization gives rise to large field intensity gradients at the vicinity of the pads. All these effects are consistent with previous calculations performed near 3D-surface protrusions $[10,16,20]$. The same situation is investigated in Fig. 4 with our DSIEM numerical code, where we used a $5 \times 5 \times 5 \mathrm{~nm}^{3}$ discretization grid for the surface protrusions. Comparison with the results of Fig. 3 shows that DSIEM provides a stronger contrast for both polarizations (e.g., for TM polarization, the square section of each pad is well reproduced).

The convergence of the RSPM method is assessed in Fig. 5. The relative total field intensity $I / I_{0}$ is computed along a scan line crossing the center of the structure (dashed line in the inset) with, respectively, 32 harmonics (long-dashed line) and 96 harmonics (dashed line). For a higher number of harmonics than 96, the result does not change. The total field intensity is computed at a height $H=20 \mathrm{~nm}$ and normalized to the value $I_{0}$ computed for a perfectly flat surface (without
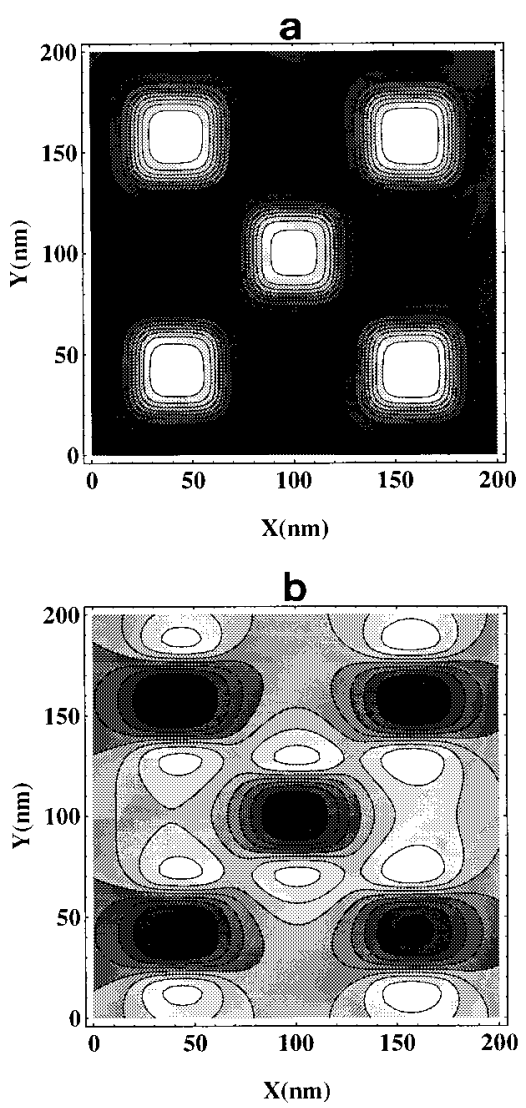

FIG. 4. Same situation as in Fig. 3, but computed with the DSIEM, using a $5 \times 5 \times 5 \mathrm{~nm}^{3}$ discretization grid for the surface protrusions.

protrusions) in TM incident polarization. The same problem is investigated in Fig. 6, but with the DSIEM approach. As the convergence of this method is extremely rapid for such small surface protrusions, we considered in this calculation surface pads with $h=20 \mathrm{~nm}$ [instead of $h=10 \mathrm{~nm}$ in Fig. 5] and the field intensity was computed at a height $H=30 \mathrm{~nm}$ above the surface. In this manner, we were able to use an extremely rough grid for the discretization: $20 \times 20 \times 20 \mathrm{~nm}^{3}$ (i.e., only four meshes per pad) (continuous line). The results obtained with this grid are already extremely good and almost indistinguishable in the intermediate region from the results obtained with a much finer grid $\left(5 \times 5 \times 5 \mathrm{~nm}^{3}\right.$, dashed line). Just above the pad the agreement is not very good, as the rough grid leads to a depletion of the field just at the center of the pad side. This depletion is simply the manifestation of the two meshes used to discretized the pad. Note also that the enhancement factor (i.e., the amplification of the field above the pad) is much more important in this figure than in Fig. 5; this is due to the larger volume of the surface protrusion in the present case.

In order to get more insight into the numerical results supplied by the two methods investigated in this paper, we give in Figs. 7 and 8 a comparison of the relative total field intensity $I / I_{0}$ scans obtained with the RSPM (continuous line) and the DSIEM (dashed line). The scans are performed along the center of the structure (dashed line in the inset), at a height $H=20 \mathrm{~nm}$ and normalized to the value $I_{0}$ computed for a perfectly flat surface (without protrusions). For RSPM, 192 harmonics were used, whereas a $5 \times 5 \times 5 \mathrm{~nm}^{3}$ discreti- 


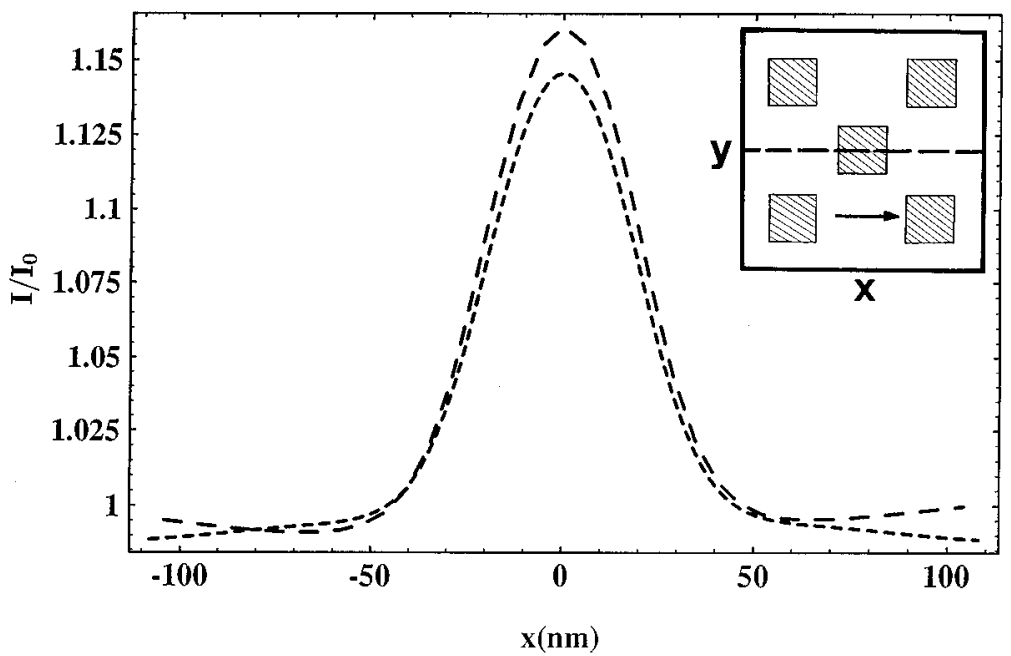

FIG. 5. Convergence study of the RSPM method. The relative total field intensity $I / I_{0}$ computed along a scanning line at the center of the structure (dashed line in the inset) with, respectively, 32 harmonics (long-dashed line) and 96 harmonics (dashed line).

zation was used for DSIEM. We note the extremely good qualitative agreement between both methods. In the TM mode (Fig. 7), DSIEM gives nonetheless a stronger contrast and a steeper intensity profile than RSPM. This is easily understood if one remembers that RSPM is based on a plane wave expansion, which is less appropriate to reproduce edges and corner profiles. We remark also the field confinement above the surface defect and a small depletion just outside the defect. In the TE mode (Fig. 8), the field profile is now smoother than in Fig. 7 and the quantitative agreement between both methods is excellent. This result again emphasizes the ability of RSPM to accurately reproduce smooth field profiles associated with subwavelength objects. Nevertheless, the first Born approximation implicitly incluced in the RSPM scheme described in Sec. III may be insufficient for investigating the optical near-field distributions spawned by more extended objects. In this case, it will be necessary to

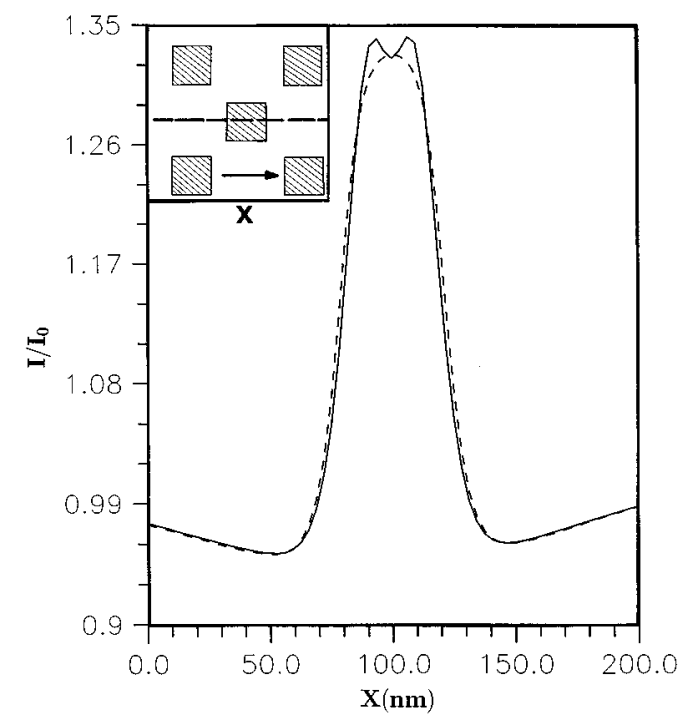

FIG. 6. Convergence study of the the DSIEM approach. We considered for this analysis surface pads with $h=20 \mathrm{~nm}$ and the field intensity was computed at a height $H=30 \mathrm{~nm}$ above the surface. Discretization grid $20 \times 20 \times 20 \mathrm{~nm}^{3}$ (i.e., only four meshes per pad) (continuous line). Discretization grid $5 \times 5 \times 5 \mathrm{~nm}^{3}$ (dashed line). solve the problem by including in Eq. (14) nonlinear higherorder terms with respect to the corrugation profile.

\section{CONCLUSION}

In the preceding section, we have observed a very good qualitative agreement, for the system under study, between the near fields computed using RSPM and DSIEM. The convergence of DSIEM is extremely rapid and a satisfactory representation of the field can be obtained even with a rough grid, although this representation cannot reach in the very near field a higher resolution than the grid used for the discretization (see, e.g., the continuous line in Fig. 6). The RSPM is particularly well suited for smooth field profiles, but encounters more difficulties for reproducing edges and straight profiles.

On the other hand, RSPM has a definite advantage for periodic structures such as grating. Indeed, a periodic structure is easily formulated in Fourier space and the evaluation

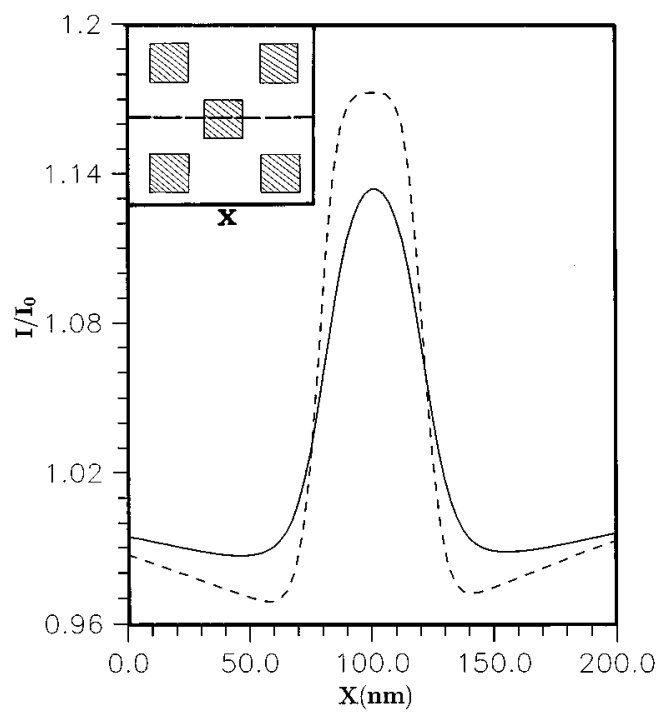

FIG. 7. Comparison of relative total field intensity $I / I_{0}$ scans obtained with the RSPM (continuous line) and the DSIEM (dashed line) for TM polarization. The scans are performed along the center of the structure (dashed line in the inset), at a height $H=20 \mathrm{~nm}$. 


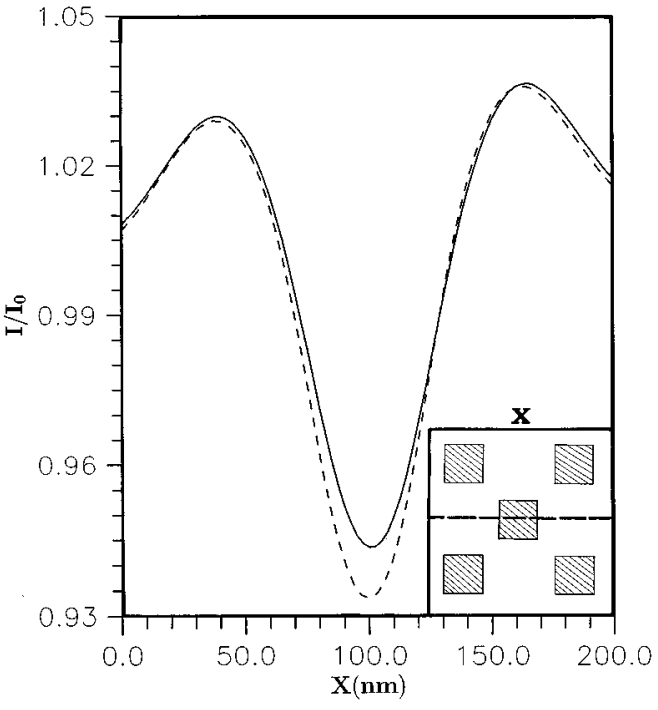

FIG. 8. Same as in Fig. 7, but for TE polarization.

of the field using Eq. (14) becomes extremely efficient. Nonetheless, for a periodic structure with a high topographic or dielectric contrast, the accuracy provided by RSPM might be insufficient. We have not considered such a periodic system in the present study because for DSIEM, the discretization of an infinite periodic structure involves a prohibitory amount of computational effort.

This actually brings up an extremely important point: the computational effort required by both methods. For small systems, similar to those investigated in the present study, both methods provide results within a few minutes on a standard desktop workstation. The main difference between the method is the scaling of the computational effort with the complexity of the problem. The RSPM requires a discretization of the surface of the system, and the computational effort grows with the square of the number of discretization points used on the entire surface. On the other hand, DSIEM requires only a discretization of the surface defects, but leads to large systems of equations that require basically a number of operations that grows with the third power of the number of meshes $N$ [more efficient schemes that require a number of operations proportional to $N^{2} \ln (N)$ can speed up the process, but at the price of some accuracy, because of the extremely high condition number of the involved matrices]. As we see, there is always in the choice of an appropriate method for NFO a tradeoff between accuracy and efficiency.

Let us emphasize that we were able to reproduce with both approaches a fundamental effect for NFO: the confinement of light by subwavelength structures. Such confined fields can be detected by a NFO local probe, which explains how a resolution much better than the diffraction limit can be
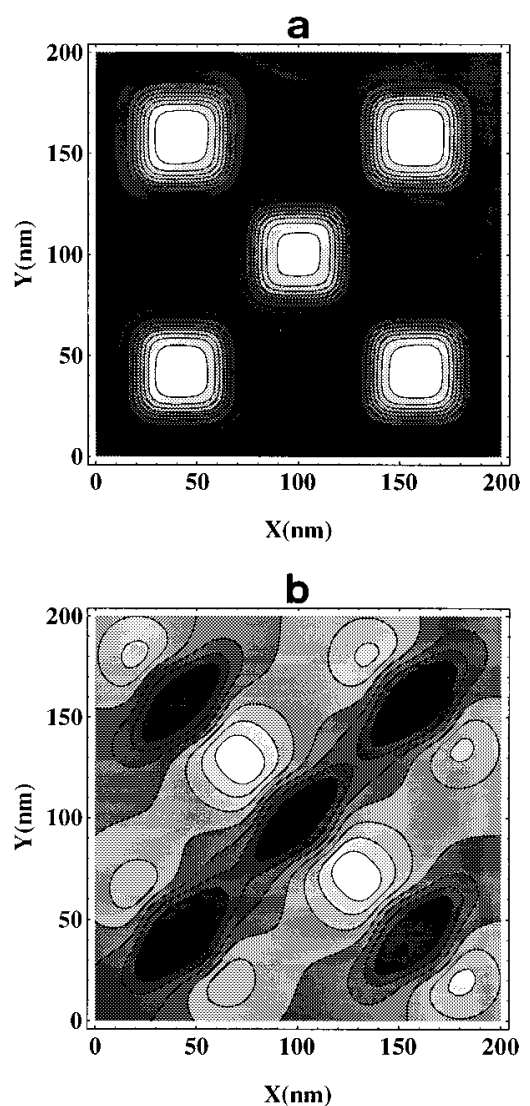

FIG. 9. Field intensity computed with DSIEM using a $5 \times 5 \times 5 \mathrm{~nm}^{3}$ discretization grid; (a) TM polarization and (b) TE polarization. The incident field propagates along the diagonal of the system $\left(\theta_{2}=45^{\circ}\right.$ in Fig. 1$)$. Note the extremely important sensibility to the polarization: for TM field, the strong field confinement dominates the total field intensity and a similar pattern is observed as for other propagation directions [compare with Fig. 4(a)]. On the other hand, for TE polarization, the total field intensity pattern strongly depends on the propagation direction and in the present figure, mainly the protrusion corners are highlighted [compare with Fig. 4(b)].

achieved in near-field microscopy. Many subtle effects are involved in NFO microscopy as is illustrated in Fig. 9. This figure emphasizes the influence of the illumination conditions on the imaging properties of subwavelength objects in near-field microscopy.

\section{ACKNOWLEDGMENTS}

We have benefited from stimulating discussions with A. Dereux, A. Castiaux, X. Bouju, and M. Spajer.
[1] V. Celli, A. Marvin, and F. Toigo, Phys. Rev. B 11, 1779 (1975).

[2] A. Marvin, F. Toigo, and V. Celli, Phys. Rev. B 11, 2777 (1975).

[3] J. M. Elson, Phys. Rev. B 12, 2541 (1975).
[4] F. Toigo, A. Marvin, V. Celli, and N. R. Hill, Phys. Rev. B 13, 5618 (1977).

[5] G. S. Agarwal, Phys. Rev. B 15, 2371 (1977).

[6] For a historical presentation of near-field optics, see, for example, D. W. Pohl, in Near Field Optics Vol. 242 of NATO 
Advanced Study Institute, Series E, edited by D. W. Pohl and D. Courjon (Kluwer, Dordrecht, 1993), pp. 1-5.

[7] D. Courjon and C. Bainier, Rep. Prog. Phys. 57, 989 (1994).

[8] Photons and Local Probes, Vol. 300 of NATO Advanced Study Institute, Series E, edited by O. Marti and R. Möller (Kluwer, Dordrecht, 1995).

[9] D. Van Labeke and D. Barchiesi, in Near-Field Optics [Ref. (6)], pp. 157-178.

[10] Ch. Girard and D. Courjon, Phys. Rev. B 42, 9340 (1990).

[11] A. Dereux, J. P. Vigneron, Ph. Lambin, and A. A. Lucas, Physica B 175, 65 (1991).

[12] D. Van Labeke and D. Barchiesi, J. Opt. Soc. Am. A 9, 732 (1992).

[13] L. Novotny, D. W. Pohl, and P. Regli, J. Opt. Soc. Am. A 11, 1768 (1994).

[14] F. Pincemin, A. Sentenac, and J.-J. Greffet, J. Opt. Soc. Am. A 11, 1117 (1994).

[15] J. P. Goudonnet, E. Bourillot, P. M. Adam, F. de Fornel, L. Salomon, P. Vincent, M. Nevière, and T. L. Ferrell, J. Opt. Soc. Am. A 12, 1749 (1995).

[16] Ch. Girard, A. Dereux, O. J. F. Martin, and M. Devel, Phys. Rev. B 50, 14467 (1994).

[17] R. Carminati, A. Madrazo, and M. Nieto-Vesperinas, Opt. Commun. 111, 26 (1994).

[18] D. Barchiesi and D. Van Labeke, Microsc. Microanal. Microstruct. 5, 435 (1994).

[19] O. J. F. Martin, A. Dereux, and Ch. Girard, J. Opt. Soc. Am. A 11, 1073 (1994).
[20] O. J. F. Martin, Ch. Girard, and A. Dereux, Phys. Rev. Lett. 74, 526 (1995).

[21] J. L. Kann, T. D. Milster, F. F. Froehlich, R. W. Ziolkowski, and J. B. Judkins, J. Opt. Soc. Am. A 12, 1677 (1995).

[22] A. Sentenac and J. J. Greffet, Ultramicroscopy 57, 246 (1995).

[23] M. Born and E. Wolf, Principles of Optics (Pergamon, New York, 1964).

[24] A. D. McLachlan, Mol. Phys. 7, 381 (1964).

[25] G. S. Agarwal, Phys. Rev. A 11, 230 (1975).

[26] H. Metiu, Prog. Surf. Sci. 17, 153 (1984).

[27] E. N. Economou, Green's Functions in Quantum Physics, 2nd ed. (Springer-Verlag, Berlin, 1990).

[28] Ch. Girard and A. Dereux, Rep. Prog. Phys. 59, 657 (1996).

[29] A. D. Yaghjian, Proc. IEEE 68, 248 (1980).

[30] R. Reddick, R. J. Warmack, and T. J. Ferrell, Phys. Rev. B 39, 767 (1989).

[31] D. Courjon, K. Sarayeddine, and M. Spajer, Opt. Commun. 71, 23 (1989).

[32] T. L. Ferrell, S. L. Sharp, and R. J. Warmack, Ultramicroscopy 42-44, 408 (1991).

[33] D. Courjon, C. Bainier, and M. Spajer, J. Vac. Sci. Technol. B 10, 2436 (1992).

[34] N. F. Van Hulst, F. B. Segerink, F. Achten, and B. Bolger, Ultramicroscopy 42-44, 416 (1992).

[35] F. de Fornel, L. Salomon, P. Adam, E. Bourillot, J. P. Goudonnet, and M. Nevière, Ultramicroscopy 42-44, 422 (1992).

[36] N. F. Van Hulst, M. H. P. Moers, and B. Bögler, J. Microsc. 171, 95 (1993). 